\title{
Searching for pathways involving dressed states in optimal control theory
}

\author{
Philipp von den Hoff,* Markus Kowalewski and Regina de Vivie- \\ Riedle
}

\author{
Received 3rd March 2011, Accepted 5th April 2011 \\ DOI: 10.1039/c1fd00031d
}

\begin{abstract}
Selective population of dressed states has been proposed as an alternative control pathway in molecular reaction dynamics [Wollenhaupt et al., J. Photochem. Photobiol. A: Chem., 2006, 180, 248]. In this article we investigate if, and under which conditions, this strong field pathway is included in the search space of optimal control theory. For our calculations we used the proposed example of the potassium dimer, in which the different target states can be reached via dressed states by resonant transition. Especially, we investigate whether the optimization algorithm is able to find the route involving the dressed states although the target state lies out of resonance in the bare state picture.
\end{abstract}

\section{Introduction}

Recent developments in ultrashort laser pulse generation and shaping technology opened the door to new strategies for the control of ultrafast molecular photoreactions. ${ }^{1,2}$ One of the novel routes uses the phase of the electric field with respect to the envelope as control parameter. ${ }^{3}$ Another approach utilizes strong electric fields to shift electronic states in energy in order to steer the molecular reactions. ${ }^{4}$ In this work we focus on the selective population of dressed states (SPODS), a strategy which nicely combines both routes of phase and strong field control. SPODS can be implemented via the photon locking technique, ${ }^{5}$ the optical counterpart to the spin locking technique, originally developed in NMR. ${ }^{6}$ The photon locking technique in combination with light field control of molecular reactions was theoretically exemplified for ground state dynamics. ${ }^{7}$ Selective population of dressed states is also the key in adiabatic radid passage (ARP) ${ }^{8}$ or stimulated Raman adiabatic passage (STIRAP). ${ }^{9,10}$ Both are adiabatic processes characterized by a smooth change in the temporal phase and enevlope of the electric field. SPODS via photon locking, on the other hand, is characterized by a rapid phase jump and is therefore of nonadiabatic nature.

Strong field quantum control via SPODS using pulse shaping techniques was experimentally demonstrated for the potassium atom by $\mathrm{M}$. Wollenhaupt and $\mathrm{T}$. Baumert. ${ }^{11}$ Within their investigations, they used sinusoidal spectral phase modulation, ${ }^{12,13}$ chirped excitation ${ }^{14}$ and adaptive optimization of the spectral phase ${ }^{11}$ to realize the SPODS scheme. They already extended the SPODS mechanism theoretically to the molecular system $\mathrm{K}_{2}{ }^{15}$ to control the final population in the $4^{1} \Sigma_{\mathrm{g}}^{+}$and $5^{1} \Sigma_{\mathrm{g}}^{+}$target states.

In a previous work we performed calculations on the SPODS in $\mathrm{K}_{2}$ to analyze the interaction of the ultrashort light field with the initialized oscillating electronic dipole moment ${ }^{16}$ as the key element for the control mechanism. As in ref. 15, we 
used a simple double pulse sequence. Depending on the parameters pulsevdelayande Online intensity, we reached a maximum efficiency of about $66 \%$ for both target states and showed the correlation between the optimal pulse delay and the lifetime of the induced electronic coherence.

In this work we shortly revisit the basics of the SPODS mechanism. With optimal control theory (OCT) we investigate whether the SPODS mechanism is an optimal solution for the given control task and - if yes - whether its efficiency can be further improved by OCT. In addition, we outline a strategy to include the SPODS mechanism in the search space of the OCT - if needed - by selecting special starting conditions.

\section{SPODS excitation scheme}

In this section we revisit the excitation scheme for the non-adiabatic SPODS mechanism for the example of $\mathrm{K}_{2}$ and summarize the previous findings. ${ }^{16}$ In the ideal case a weak and resonant pulse first creates a state of maximum coherence, i.e. a $50: 50$ superposition between the bare electronic states $\mathrm{X}^{1} \Sigma_{\mathrm{g}}^{+}$and $\mathrm{A}^{1} \Sigma_{\mathrm{u}}^{+}$(see Fig. 1 dash dotted arrow). This process simultaneously launches an oscillating dipole moment following the driving field with a phase shift of $\frac{\pi}{2}$. The formation of the superposition and the rise of the induced dipole moment following the driving laser field is schematically illustrated in Fig. 2 (a) and (b) (first 40 fs).

In the second step the pre-pulse is followed by an intense pulse with the same frequency. This pulse is shifted in phase by $\pm \frac{\pi}{2}$ relative to the first one. Thus the electric field of the second pulse is either exactly in phase with the prepared oscillating molecular dipole or exactly shifted by $\pi$ (see Fig. 2 (b). The in-phase situation selectively populates the lower dressed state (DS) (see Fig. 1 lower dotted curve), the $\pi$ shift leads to a selective population of the upper DS state (see Fig. 1 upper dotted curve). During the second pulse the bare state populations are locked due to the phase relation, preventing population transfer between the $\mathrm{X}^{1} \Sigma_{\mathrm{g}}^{+}$and $\mathrm{A}^{1} \Sigma_{\mathrm{u}}^{+}$, although

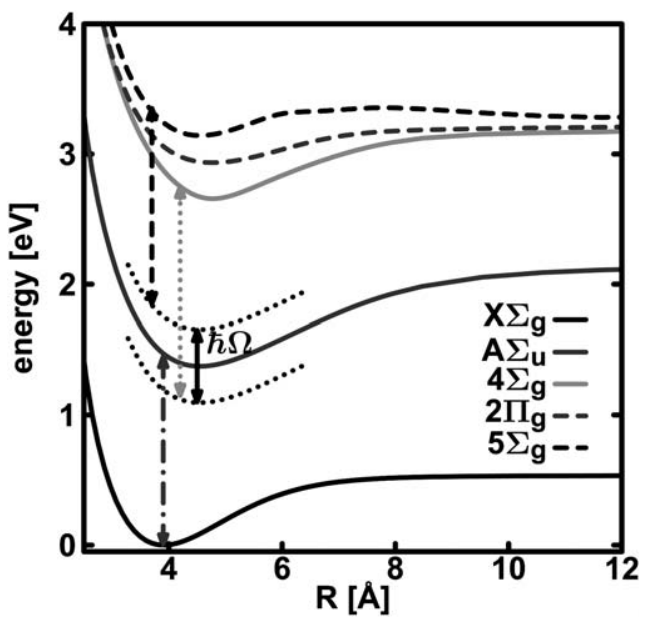

Fig. 1 SPODS scheme of potassium dimer. The first pulse in the sequence creates a superposition between the $\mathrm{X}^{1} \Sigma_{\mathrm{g}}^{+}$and $\mathrm{A}^{1} \Sigma_{\mathrm{u}}^{+}$states (dash dotted arrow). During the second pulse the $\mathrm{X}^{1} \Sigma_{\mathrm{g}}^{+}$ and the $\mathrm{A}^{1} \Sigma_{\mathrm{u}}^{+}$states are 'photon locked'. The optical phase controls which of the dressed states (indicated as dotted lines) energetically separated by $\hbar \Omega$ is selectively populated. Absorption of another photon leads to population transfer to either the $4^{1} \Sigma_{\mathrm{g}}^{+}$(dotted arrow) or $5^{1} \Sigma_{\mathrm{g}}^{+}$(dashed arrow). 

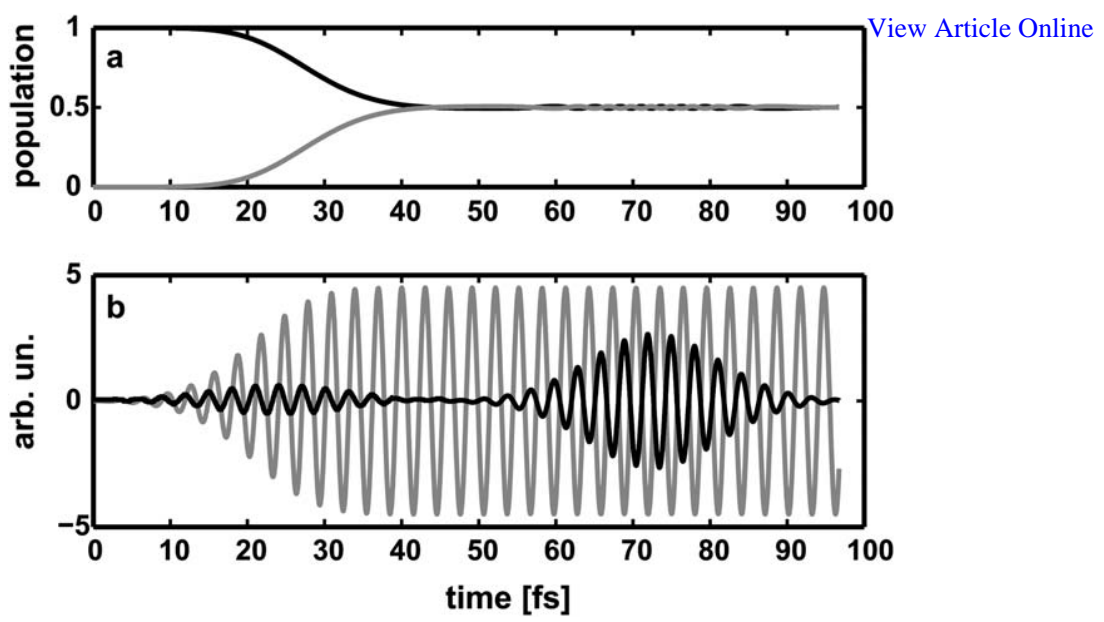

Fig. 2 Schematic illustration of the SPODS pulse sequence populating the upper dressed state acting on two electronic states. (a) Temporal evolution of the populations of the two electronic states. (b) Temporal evolution of the electric field (black) and the induced dipole moment (gray).

the frequency is resonant on this transition (see Fig. 2 (a) ). ${ }^{7}$ Due to the laser intensity dependent energy splitting of the DS in the order of $\hbar \Omega$ (solid black arrow in Fig. 1) resonance is reached either with the $4^{1} \Sigma_{\mathrm{g}}^{+}$or the $5^{1} \Sigma_{\mathrm{g}}^{+}$target state. With the described pulse sequence it is in principle possible to control the final populations in the $4^{1} \Sigma_{\mathrm{g}}^{+}$ and $5^{1} \Sigma_{\mathrm{g}}^{+}$state by switching the relative phase between the two sub-pulses.

In the case of the potassium atom it is possible to separate the two involved subpulses well in time. ${ }^{12,13}$ But in the case of the potassium dimer it turned out that the two sub-pulses have to overlap partially in time to ensure an efficient SPODS. ${ }^{15}$ To explain their results, we used our approach for the coupled electron and nuclear quantum dynamics ${ }^{17-19}$ and followed the time-dependent expectation value of the induced dipole moment along the molecular $z$-axis $\left\langle\mu_{z}\right\rangle(t)$. The value of $\left\langle\mu_{z}\right\rangle(t)$ is related to the time-dependent electron density $\varphi_{\text {tot }}(t)^{16}$ through:

$$
\left\langle\mu_{\mathrm{z}}\right\rangle(t)=\left\langle\varphi_{\text {tot }}(t)\left|\hat{\mu}_{\mathrm{z}}\right| \varphi_{\text {tot }}(t)\right\rangle=\int \rho_{\text {tot }}(t) \hat{\mu}_{\mathrm{z}} \mathrm{dr} .
$$
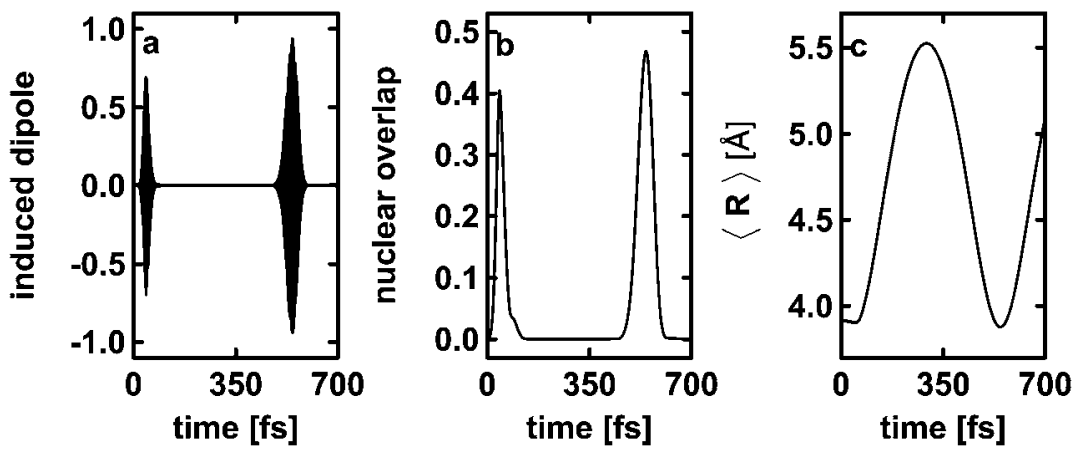

Fig. 3 Temporal evolution in the presence of the pre-pulse of (a): the expectation value of the induced dipole moment $\left\langle\mu_{\mathrm{z}}\right\rangle$; (b): the overlap of the nuclear wavefunctions on the $\mathrm{X}^{1} \Sigma_{\mathrm{g}}^{+}$and $\mathrm{A}^{1} \Sigma_{\mathrm{u}}^{+}$state; (c): the expectation value of the internuclear distance of the nuclear wavefunction on the $\mathrm{A}^{1} \Sigma_{\mathrm{u}}^{+}$state $(\langle R\rangle)$. 
$\hat{\mu}_{\mathrm{z}}$ is the dipole operator along the intramolecular axis. Fig. 3 (a) shows thexexpecte Online tation value of the induced dipole moment in the presence of the pre-pulse alone. The corresponding overlap of the nuclear wavefunctions on the $\mathrm{X}^{1} \Sigma_{\mathrm{g}}^{+}$and the $\mathrm{A}^{1} \Sigma_{\mathrm{u}}^{+}$surface is plotted in Fig. 3 (b). Both observables reflect the formation of the superposition between the $\mathrm{X}^{1} \Sigma_{\mathrm{g}}^{+}$and $\mathrm{A}^{1} \Sigma_{\mathrm{u}}^{+}$state within the first $40 \mathrm{fs}$. Right after the end of the pulse both values begin to decrease in the same manner, although the populations of the involved sates stay constant. The reason for this decay lies in the nuclear wavepacket dynamics. After the pre-pulse, the two nuclear wavepackets start to evolve differently, due to the shift of the $\mathrm{A}^{1} \Sigma_{\mathrm{u}}^{+}$state towards larger internuclear distances and the difference in potential shape (see Fig. 1). In addition only large amplitude motion is induced in the upper potential, while the wavepacket in the ground state nearly stays fixed in space. This extended nuclear motion can be seen in the time-dependent expectation value of the internuclear distance $\langle R\rangle(t)$ for the nuclear wavepacket in the $\mathrm{A}^{1} \Sigma_{\mathrm{u}}^{+}$state (see Fig. 3 (c)).

Now the reason for the partial temporal overlap of the two sub-pulses becomes obvious. As the nuclear wavepackets start to evolve asynchronously, the induced dipole moment is damped by the reducing overlap of the two nuclear wavepackets. As a consequence the interaction between the induced dipole moment and the second pulse (i.e. the photon locking) gets less effective and leads to the loss of control. In the potassium atom this effect is absent due to the non-existing nuclear dynamics. In Fig. 3 (a) a revival of the expectation value of the induced dipole moment with even an increase in amplitude can be seen after one oscillation period of the nuclear wavepacket in the upper potential (after approx. $500 \mathrm{fs}$ ). From the theory, this revival can also be used to continue the SPODS mechanism with a long pulse delay of about $550 \mathrm{fs}$.

\section{Optimal control theory}

OCT is established as a powerful method for various control tasks and for the interpretation of the induced control mechanisms. Different schemes for quantum control investigations were developed based on the calculus of variations. ${ }^{20-22}$ The OCT algorithm is used to find the optimal laser pulse, driving a quantum system from a defined initial state $\psi(t=0)$ to the wanted target state $\Phi(t=T) .{ }^{23,24}$ For the optimization of the SPODS mechanism, the final population of the target state needs to be optimized. Therefore we used the expectation value of a positive definite operator as control $\mathrm{aim}^{21}$ in the following form: ${ }^{25}$

$$
\begin{aligned}
J[\psi(t), \Phi(t), \varepsilon(t)]= & |\langle\psi(T)|\hat{O}| \psi(T)\rangle|^{2}-\int_{0}^{T} \alpha(t)\left|\varepsilon(t)-\varepsilon_{\mathrm{ref}}(t)\right|^{2} d t \\
& -4 \Re\left[\langle\psi(T)|\hat{O}| \psi(T)\rangle \int_{0}^{T}\left\langle\Phi(t)\left|\left[\frac{i}{\hbar}\left(\hat{H}_{0}-\hat{\mu} \varepsilon(t)\right)+\frac{\partial}{\partial t}\right]\right| \psi(t)\right\rangle d t\right]
\end{aligned}
$$

It includes three terms, the optimization target, an integral over the laser field, penalizing the pulse fluence and the time dependent Schrödinger equation as a side condition. The optimization target is to maximize the absolute square of the expectation value of any positive definite operator $|\langle\psi(T)|\hat{O}| \psi(T)\rangle|^{2}$ using the wavefunction $\psi$ after the laser excitation time $T$. The initial states can be chosen as eigenstates or arbitrary superpositions of eigenstates. The second term of eqn (2) is an integral over the laser field $\varepsilon(t)$ and a reference field $\varepsilon_{\text {ref }}(t)$ with a time-dependent factor $\alpha(t)$. Depending on the implementation of $\varepsilon_{\text {ref }}(t)$, it is known as the penalty factor or Krotov change parameter. With the choice of $\alpha(t)=\alpha_{0} / s(t)$ and e.g. a gaussian shaped function $s(t)$, an field envelope function can be imprinted on the laser field. ${ }^{26,27}$ This envelope guarantees smooth on and off switching of the pulse for the times $t=0$ and $t=T$. The last term of the functional (eqn (2)) comprises the time-dependent Schrödinger equation as an additional constraint. 
The time independent part of the molecular Hamiltonian is denoted by $H_{0}$ VieheAarsere Online field couples to the dipole moment with the interaction term $-\hat{\mu} \varepsilon(t)$.

The calculation of optimal laser fields now relies on finding the extremal value of the functional $J$ with respect to the functions $\psi(t), \Phi(t)$ and $\varepsilon(t)$. Separable differential equations can be derived ${ }^{28}$ which involve the propagation of the initial state $\psi(0)$, the target state $\Phi(T)=\hat{O} \psi(T)$ and an equation for the electric field. The equations are solved iteratively with the Krotov method. ${ }^{28,29}$ The laser field for the next iteration $n+1$ can be formulated as:

$$
\varepsilon^{n+1}(t)=\varepsilon^{n}(t)+\frac{s(t)}{2 \alpha} \mathfrak{S}\left[\left\langle\psi\left(T, \varepsilon^{n+1}\right)|\hat{O}| \psi\left(T, \varepsilon^{n}\right)\right\rangle\left\langle\psi\left(t, \varepsilon^{n}\right)|\hat{\mu}| \psi\left(t, \varepsilon^{n+1}\right)\right\rangle\right] .
$$

with $\varepsilon^{n}=\varepsilon_{\text {ref }}$

\section{The SPODS benchmark for the OCT calculations}

To set a benchmark for the OCT algorithm, we first performed calculations using a time dependent electric field $\varepsilon(t)$ representing a double pulse of the form:

$$
\begin{aligned}
\varepsilon(t)= & E_{1} e^{-2 \ln 2\left(\frac{\left(t-t_{1}\right)}{\sigma}\right)^{2}} \cos \left(\omega \cdot\left(t-t_{1}\right)\right) \\
& +E_{2} e^{-2 \ln 2\left(\frac{\left(t-t_{2}\right)}{\sigma}\right)^{2}} \cos \left(\omega \cdot\left(t-t_{2}\right)+\phi\right) .
\end{aligned}
$$

Here $E_{1,2}$ denote the maximum electric fields of the two sub-pulses centered in time at $t_{1}, 2$. Both pulses have the same frequency $\omega$ and full width at half maximum (FWHM) $\sigma=20 \mathrm{fs}$. The second pulse holds a phase $\phi$. By setting the parameters $\Delta T=t_{2}-t_{1}$ and $\phi$ to specific values, one can obtain phase shifts of $\pm \frac{\pi}{2}$. For the technical details on the calculation of the potential energy surfaces and on the wavepacket propagation see ref. 16 .

First we optimized the maximum electric field of the pre-pulse $E_{1}$ to reach the state of maximum coherence. Subsequently we systematically varied the maximum electric field $E_{2}$ and $\Delta T$. The final population in the individual target state is monitored. In order to conserve phase shifts of $\pm \frac{\pi}{2}$ we used a constant $\phi= \pm \frac{\pi}{2}$ and varied the time delay in integer multiples of the oscillation period of the driving field $(\omega=$ 0.05392 a.u. $=845 \mathrm{~nm} ; 1$ period $=2.8 \mathrm{fs})$. For both target states we scanned $E_{2}$ values ranging from 0.005 to $0.055 \mathrm{GV} \mathrm{cm}^{-1}$ and $\Delta T$ values between 1 and 15 periods.

A maximum efficiency for the SPODS scheme of about $66 \%$ for both target states was achieved. The optimized parameters are given in Table 1 . The resulting population dynamics, together with the optimized laser pulses and the pulse characterizations are summarized in Fig. 4 and 5. The upper panels show the population dynamics of the electric states involved for the $4^{1} \Sigma_{\mathrm{g}}^{+}$(Fig. 4) and $5^{1} \Sigma_{\mathrm{g}}^{+}$(Fig. 5) target

Table 1 Optimized laser parameters for population of the $4^{1} \Sigma_{\mathrm{g}}^{+}$and $5^{1} \Sigma_{\mathrm{g}}^{+}$target state

\begin{tabular}{lll}
\hline target state & $4^{1} \Sigma_{\mathrm{g}}^{+}$ & $5^{1} \Sigma_{\mathrm{g}}^{+}$ \\
\hline$E_{1}\left[\mathrm{GV} \mathrm{cm}^{-1}\right]$ & 0.002 & 0.002 \\
$E_{2}\left[\mathrm{GV} \mathrm{cm}^{-1}\right]$ & 0.048 & 0.036 \\
$\Delta \mathrm{T}\left[\right.$ periods $\left.^{a}\right]$ & 5 & 5 \\
${ }^{a} 5$ periods correspond to $14 \mathrm{fs}$. & &
\end{tabular}




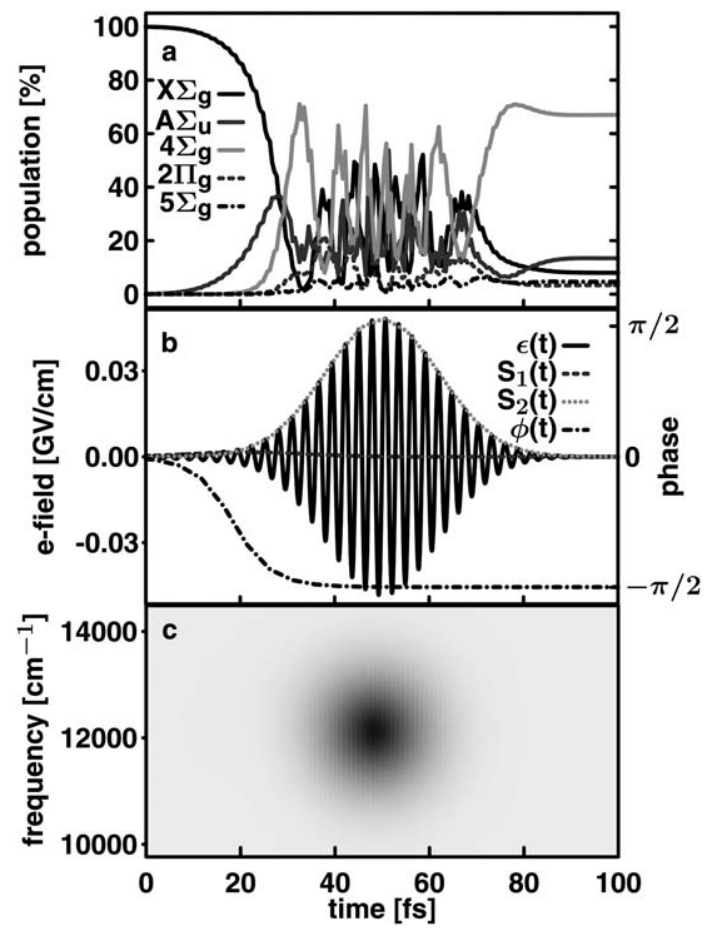

Fig. 4 Population dynamics, laser pulses and the pulse characterizations for optimized SPODS mechanisms. (a): Temporal evolution of the population in the electronic states involved. (b): Pulse sequence $\left(\varepsilon(t)\right.$; solid line) for the population of the $4^{1} \Sigma_{\mathrm{g}}^{+}$target state is plotted together with the envelopes of the pre-pulse $\left(\mathrm{S}_{1}\right.$; dashed line) and the second pulse $\left(\mathrm{S}_{2}\right.$; dotted line). The phase shift between the two pulses can be seen in the temporal phase $\phi$ $(t)$ (dashed dotted line; right ordinate). (c): Cross-correlated frequency-resolved optical gating (XFROG) diagrams of the pulse sequence.

state. Both graphs show the build up of the superposition between the $\mathrm{X}^{1} \Sigma_{\mathrm{g}}^{+}$and the $\mathrm{A}^{1} \Sigma_{\mathrm{u}}^{+}$within the first $30 \mathrm{fs}$. After $30 \mathrm{fs}$ the temporal phase $\phi(t)$ of the laser field has changed (phase shift $-\frac{\pi}{2}$; see Fig. 4 dash dotted line; phase shift $\frac{\pi}{2}$; see Fig. 4 dash dotted line) and both population dynamics proceed differently. In the case of the $-\frac{\pi}{2}$ phase shift (Fig. 4 (a)) the population is transferred form the superposition via the lower DS to the $4^{1} \Sigma_{\mathrm{g}}^{+}$target state (solid light gray line), while in the case of the $\frac{\pi}{2}$ phase shift (Fig. 5 (a)) the population is transferred from the superposition via the upper DS to the $5^{1} \Sigma_{\mathrm{g}}^{+}$target state (black dashed dotted line). The results in Fig. 4 and 5 demonstrate, that the control task is realized by only changing the sign of the interpulse phase shift and by adjusting the intensity of the second pulse. The overall structure of the two optimized pulses stays identical, as shown in the cross-correlated frequency-resolved optical gating (XFROG) representations (Fig. 4 (c) and 5 (c)).

\section{Optimization of the SPODS mechanism using OCT}

As target definition for OCT we use the absolute square of the expectation value of an operator $|\langle\psi(T)|\hat{O}| \psi(T)\rangle|^{2}$. To give the algorithm as much flexibility as possible we used projection operators $\hat{O}$, projecting the nuclear wavefunction 


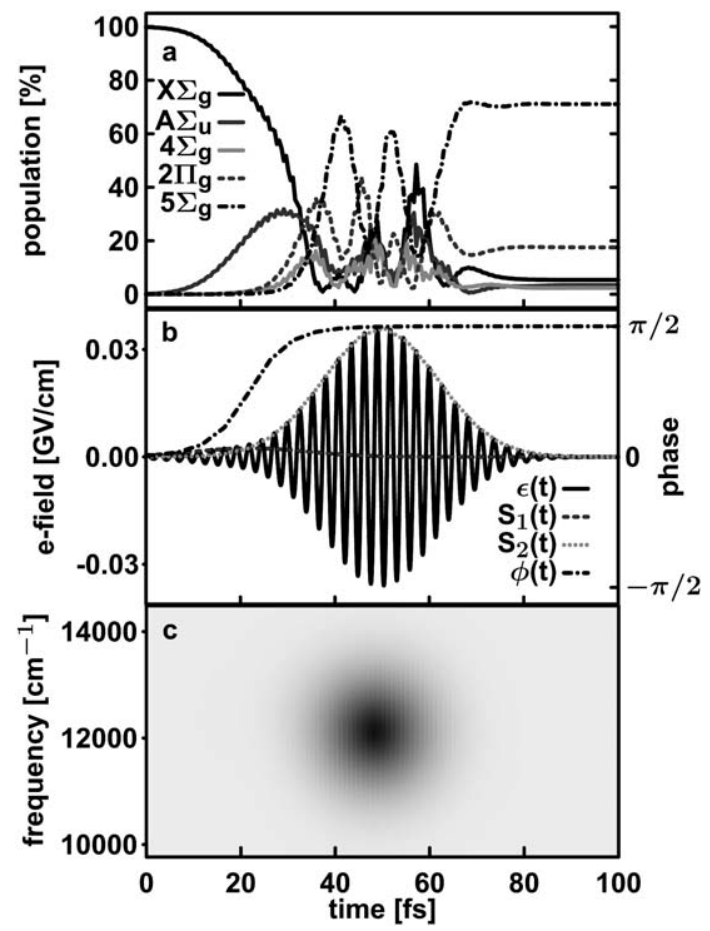

View Article Online

Fig. 5 Population dynamics, laser pulses and the pulse characterizations for optimized SPODS mechanisms. (a): Temporal evolution of the population in the electronic states involved. (b): Pulse sequence $\left(\varepsilon(t)\right.$; solid line) for the population of the $5^{1} \Sigma_{\mathrm{g}}^{+}$target state is plotted together with the envelopes of the pre-pulse $\left(S_{1}\right.$; dashed line) and the second pulse $\left(\mathrm{S}_{2}\right.$; dotted line). The phase shift between the two pulses can be seen in the temporal phase $\phi$ $(t)$ (dashed dotted line; right ordinate). (c): Cross-correlated frequency-resolved optical gating (XFROG) diagrams of the pulse sequence.

$$
\psi(t)=\left(\begin{array}{c}
\chi_{\mathrm{X}^{1} \Sigma_{\mathrm{g}}^{+}}(t) \\
\chi_{\mathrm{A}^{1} \Sigma_{\mathrm{u}}^{+}}(t) \\
\chi_{4^{1} \Sigma_{\mathrm{g}}^{+}}(t) \\
\chi_{2^{1} \Pi_{\mathrm{g}}^{+}}(t) \\
\chi_{5^{1} \Sigma_{\mathrm{g}}^{+}}(t)
\end{array}\right)
$$

on the target electronic state e.g. the $4^{1} \Sigma_{\mathrm{g}}^{+}\left(\hat{O}_{4^{1} \Sigma_{\mathrm{g}}^{+}}\right)$or $5^{1} \Sigma_{\mathrm{g}}^{+}\left(\hat{O}_{5^{1} \Sigma_{\mathrm{g}}^{+}}\right)$state:

$$
\hat{O}_{4^{1} \Sigma_{\mathrm{g}}^{+}}=\left(\begin{array}{ccccc}
0 & 0 & 0 & 0 & 0 \\
0 & 0 & 0 & 0 & 0 \\
0 & 0 & 1 & 0 & 0 \\
0 & 0 & 0 & 0 & 0 \\
0 & 0 & 0 & 0 & 0
\end{array}\right) \quad \text { and } \hat{O}_{5^{1} \Sigma_{\mathrm{g}}^{+}}=\left(\begin{array}{ccccc}
0 & 0 & 0 & 0 & 0 \\
0 & 0 & 0 & 0 & 0 \\
0 & 0 & 0 & 0 & 0 \\
0 & 0 & 0 & 0 & 0 \\
0 & 0 & 0 & 0 & 1
\end{array}\right) \text {. }
$$

These projection operators (eqn (6)) make the target independent from the spatial shape of the wavefunction. In agreement with SPODS only the final population of the target state is decisive. Thus additional constraints on the final wavefunction are avoided. 


\subsection{Optimization of the $4^{1} \Sigma_{\mathrm{g}}^{+}$target using OCT}

We started the OCT calculations with optimization for the $4{ }^{1} \Sigma_{\mathrm{g}}^{+}$state. For the initial laser field $\varepsilon^{0}(t)$ we used a gaussian shaped pulse with a central frequency $\omega=911 \mathrm{~nm}$, a full width at half maximum FWHM $=20$ fs and a maximum electric field $E_{\max }=$ $0.0026 \mathrm{GV} \mathrm{cm}^{-1}$. The field strength was chosen in order to start the algorithm in the weak field regime. The frequency and the FWHM was chosen in order to include the $\mathrm{X}^{1} \Sigma_{\mathrm{g}}^{+}$to $\mathrm{A}^{1} \Sigma_{\mathrm{u}}^{+}$and simultaneously the $\mathrm{A}^{1} \Sigma_{\mathrm{u}}^{+}$to $4^{1} \Sigma_{\mathrm{g}}^{+}$transition within the frequency spectrum of the pulse but to exclude the $\mathrm{A}^{1} \Sigma_{\mathrm{u}}^{+}$to $5^{1} \Sigma_{\mathrm{g}}^{+}$transition. The initial laser field $\varepsilon^{0}(t)$ and the corresponding temporal evolution of the population in the electronic states involved are shown in Fig. 6 (a) and (b). From panel (b) it becomes obvious, that the initial field already populates the $4^{1} \Sigma_{\mathrm{g}}^{+}$target state up to $4 \%$.

The optimization is performed using the OCT algorithm with a given time span of $T=75 \mathrm{fs}$, a Krotov change parameter $\alpha_{0}=1$ a.u. and the initial Gaussian shaped pulse described above (see Fig. 6 (a)). The algorithm yields a highly efficient laser field which transfers about $98.3 \%$ from the electronic and vibrational ground state to the $4^{1} \Sigma_{\mathrm{g}}^{+}$target state. The induced population dynamics, the optimized laser pulse and the pulse characterization are shown in Fig. 7. To analyze the resulting pulse sequence and to verify whether the population dynamics follow the SPODS scheme we extracted the temporal phase $\phi(t)$ from the optimized electric field $\varepsilon(t)$. We followed the idea of ref. 30 and used the complex representation of the electric field in the time domain $\tilde{\varepsilon}^{+}(t)$ (the tilde denotes complex values) which delivers only positive frequencies after Fourier transformation (FT):

$$
\begin{aligned}
& \tilde{\varepsilon}^{+}(t)=F T^{-1}\left\{\tilde{\varepsilon}^{+}(\Omega)\right\}=\frac{1}{2 \pi} \int_{-\infty}^{\infty} \tilde{\varepsilon}^{+}(\Omega) e^{i \Omega t} \mathrm{~d} \Omega \\
& \text { with } \tilde{\varepsilon}^{+}(\Omega)=|\tilde{\varepsilon}(\Omega)| e^{i \Phi(\Omega)}= \begin{cases}\tilde{\varepsilon}(\Omega) & \text { for } \Omega \geq 0 \\
0 & \text { for } \Omega<0\end{cases} \\
& \text { and } \tilde{\varepsilon}(\Omega)=F T\{\varepsilon(t)\}=\int_{-\infty}^{\infty} \varepsilon(t) e^{-i \Omega t} \mathrm{~d} t .
\end{aligned}
$$

From $\tilde{\varepsilon}+(t)$ the temporal phase can be calculated as:

$$
\tilde{\varepsilon}^{+}(t)=\frac{1}{2} S(t) \mathrm{e}^{i \Gamma(t)}=\frac{1}{2} S(t) \mathrm{e}^{i \phi(t)} \mathrm{e}^{i \omega_{l} t} .
$$

Here $S(t)$ is the field envelope, $\omega_{l}$ the central frequency of the optimized electric field $\hat{\varepsilon}(t)$ (in this case $11600 \mathrm{~cm}^{-1}(860 \mathrm{~nm})$ see Fig. 7 (c)). The extracted temporal phase $\phi(t)$ (dash dotted line in Fig. 7 (b); right ordinate) clearly shows a jump of $-\frac{\pi}{2}$ in the time interval between 15 and $20 \mathrm{fs}$. Right after this phase jump the $4^{1} \Sigma_{\mathrm{g}}^{+}$target state starts to be populated form the initially prepared superposition between the $\mathrm{X}^{1} \Sigma_{\mathrm{g}}^{+}$and the $\mathrm{A}^{1} \Sigma_{\mathrm{u}}^{+}$state.

This population dynamics, together with the temporal phase and the shape of the pulse sequence, is very similar to those observed in Sec. 4 but with markedly higher efficiency. Thus we conclude, that the OCT algorithm has found the SPODS scheme as the optimal path to selectively populate the $4^{1} \Sigma_{\mathrm{g}}^{+}$target state within the given boundary and starting conditions.

\subsection{Optimization of the $5^{1} \Sigma_{\mathrm{g}}^{+}$target using OCT}

To optimize the selective population of the $5^{1} \Sigma_{\mathrm{g}}^{+}$target state by OCT, we cannot use the same initial laser field as in Sec. 5.1 because the target state is not accessible by this electric field. As a consequence the optimization fails or gets at least extremely inefficient. But from SPODS we know, that changing the phase shift from $-\frac{\pi}{2}$ to $\frac{\pi}{2}$ 


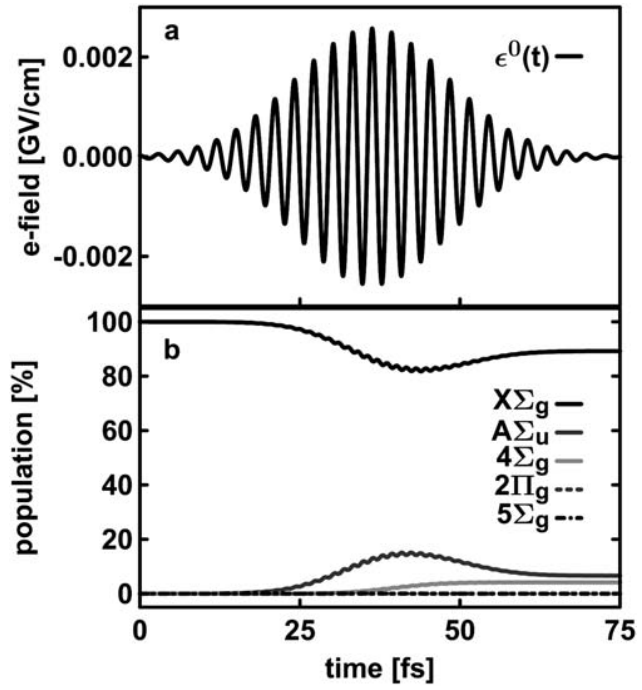

Fig. 6 Population dynamics using the initial laser field, starting the OCT algorithm. (a) Initial electric field $\varepsilon^{0}(t)$. (b) Temporal evolution of the population in the electronic states involved.

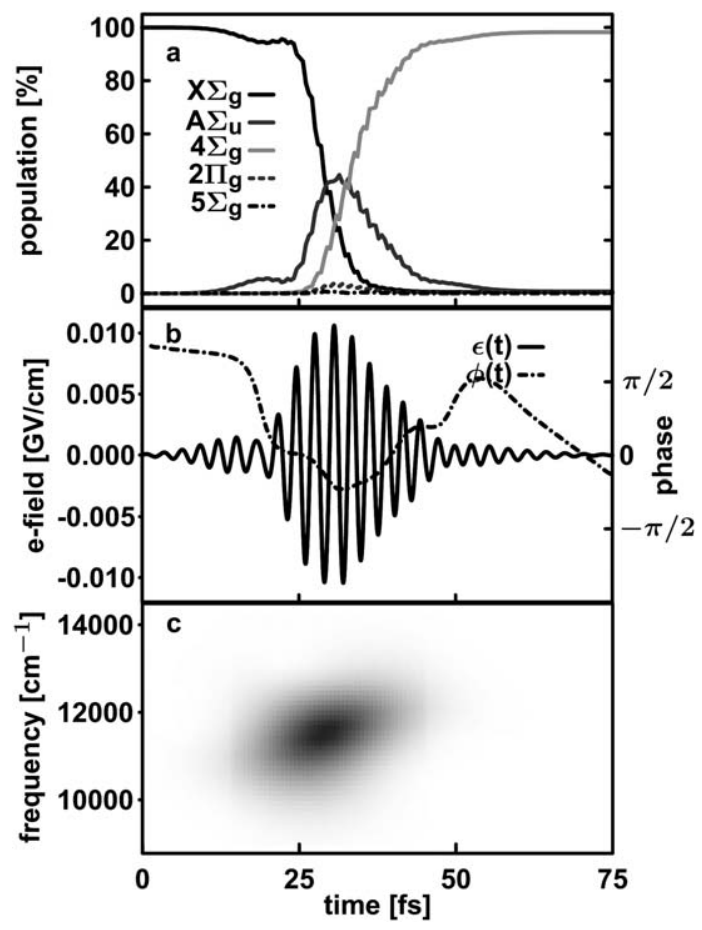

Fig. 7 Population dynamics, laser pulse and pulse characterization for the optimized SPODS mechanism. (a) Temporal evolution of the population in the electronic states involved. (b) Optimized pulse sequence for the selective population of the $4^{1} \Sigma_{\mathrm{g}}^{+}$target state $\phi(t)$ (solid line). The phase shift between the two pulses can be seen in the temporal phase $(\phi(t)$; dashed dotted line; right ordinate). (c) XFROG diagrams of the pulse sequence. 
leads to a selective population of the upper DS, making the $5^{1} \Sigma_{\mathrm{g}}^{+}$state deessibilete Online Thus we inverted the sign of the temporal phase of the optimized electric field (from Sec. 5.1) and reconstructed the corresponding laser field, now exhibiting a phase shift of $\frac{\pi}{2}$. The reconstruction was done by reversing the steps used to extract the temporal phase, but with the inverted sign of $\phi(t)$. Both pulses, the optimized $(\varepsilon$ $(t))$ and the reconstructed pulse $\left(\varepsilon_{\mathrm{r}}(t)\right)$, together with their corresponding temporal phase $\left(\phi(t)\right.$ and $\left.\phi_{\mathrm{r}}(t)\right)$ are shown in Fig. 8 (a). Here the overall phase shift of $\pi$ between the two pulses can be seen directly in the electric fields. The population dynamics, using the reconstructed electric field, is plotted in Fig. 8 (b) and clearly shows the switching between the DS. After the phase shift of $\frac{\pi}{2}$ (after approx. 25 fs) now all three electronic states (i.e. the $4^{1} \Sigma_{\mathrm{g}}^{+}$, the $2^{1} \Pi^{+}{ }_{g}$ and the $5^{1} \Sigma_{\mathrm{g}}^{+}$) are accessible from the upper DS within the spectral width of the laser pulse. As a result we now found a population of $6.4 \%$ in the $5^{1} \Sigma_{\mathrm{g}}^{+}$state and a significantly reduced population of $20.8 \%$ in the $4^{1} \Sigma_{\mathrm{g}}^{+}$. This behavior emphasizes, that the OCT algorithm has found the SPODS mechanisms.

The further optimization for the $5^{1} \Sigma_{\mathrm{g}}^{+}$target state is performed using the OCT algorithm with the same time span as in Sec. $5.1(T=75 \mathrm{fs})$, a Krotov change parameter $\alpha_{0}=1$ a.u. and the reconstructed electric field $\varepsilon_{\mathrm{r}}(t)$ exhibiting a phase shift of $\frac{\pi}{2}$ (see Fig. 8 (a)). Again the OCT algorithm yields a highly efficient laser field which transfers of about $96.7 \%$ from the electronic and vibrational ground state to the $5^{1} \Sigma_{\mathrm{g}}^{+}$target state. The resulting population dynamics, the optimized laser pulse and the pulse characterization are summarized in Fig. 9. The temporal evolution of the population in the states involved (panel (a)) again show the build up of the superposition between the $\mathrm{X}^{1} \Sigma_{\mathrm{g}}^{+}$state and the $\mathrm{A}^{1} \Sigma_{\mathrm{u}}^{+}$in the first $25 \mathrm{fs}$. Right after the phase jump of $\frac{\pi}{2}$ (Fig. 9 (b) dash dotted line; $11800 \mathrm{~cm}^{-1}(844 \mathrm{~nm})$ ) in the time interval between 15 and $25 \mathrm{fs}$, the $5^{1} \Sigma_{\mathrm{g}}^{+}$target state gets populated up to the final value.

The comparison between the OCT solutions and the manually optimized SPODS sequences (see Sec. 4) show that the OCT algorithm uses additional control knobs

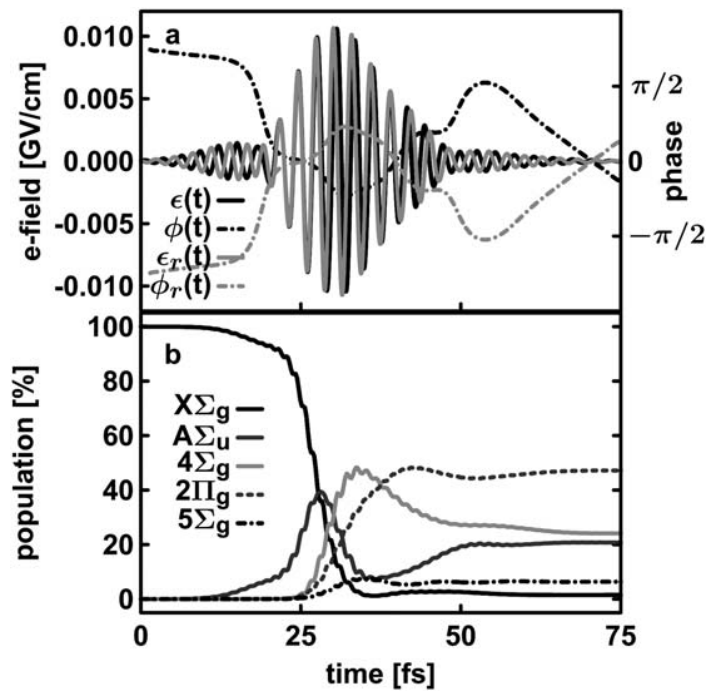

Fig. 8 (a) Optimized pulse sequence for the selective population of the $4^{1} \Sigma_{\mathrm{g}}^{+} \operatorname{target}$ state $(\varepsilon(t)$; gray solid line) and the corresponding temporal phase $(\phi(t)$; gray dash dotted line). Pulse sequence $\left(\varepsilon_{\mathrm{r}}(t)\right.$; black solid line) with reversed temporal phase $\left(\phi_{\mathrm{r}}(t)\right.$; black dash dotted line). (b) Temporal evolution of the population in the electronic states involved propagated with $\varepsilon_{\mathrm{r}}(t)$. 


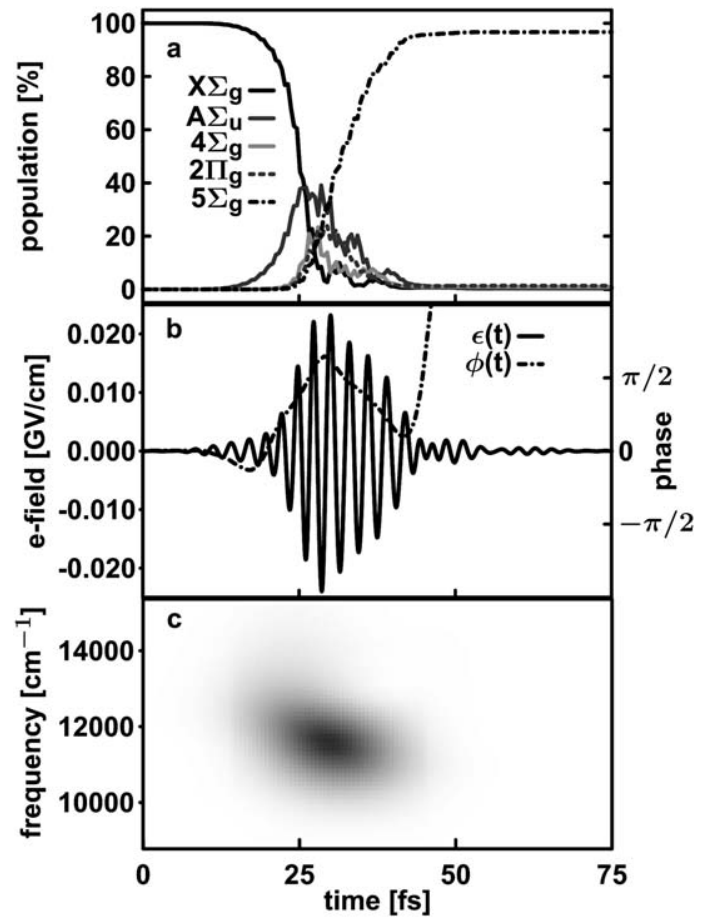

Fig. 9 Population dynamics, laser pulses and the pulse characterizations for optimized SPODS mechanisms. (a) Temporal evolution of the population in the electronic states involved. (b) Pulse sequence for the selective population of the $5^{1} \Sigma_{\mathrm{g}}^{+}$target state $(\varepsilon(t)$; solid line). The phase shift between the two pulses can be seen in the temporal phase $(\phi(t)$; dashed dotted line; right ordinate). (c) XFROG diagrams of the pulse sequence.

like chirp to allow efficient adiabatic transitions to the target states by keeping the laser intensity as low as possible (see Fig. 4 (c), and 5 (c), 7 (c) and 9 (c)). The direct comparison between the two optimized laser fields $\left(\varepsilon^{4^{1} \Sigma_{\mathrm{g}}^{+}}(t)\right.$ and $\left.\varepsilon^{5^{1} \Sigma_{\mathrm{g}}^{+}}(t)\right)$ is shown in Fig. 10. Here the two most prominent differences become obvious. The first is the $\pi$ phase shift between the pulses in the time interval from 0 to $25 \mathrm{fs}$, while the two pulses are nearly in phase in the subsequent interval between 25 and $45 \mathrm{fs}$. This phase shift proves, that the control of both target states achieved by the OCT follow the SPODS scheme. The short pulse duration of the optimized pulses with the fast varying envelope underlines the non-adiabacity of the process. The second difference

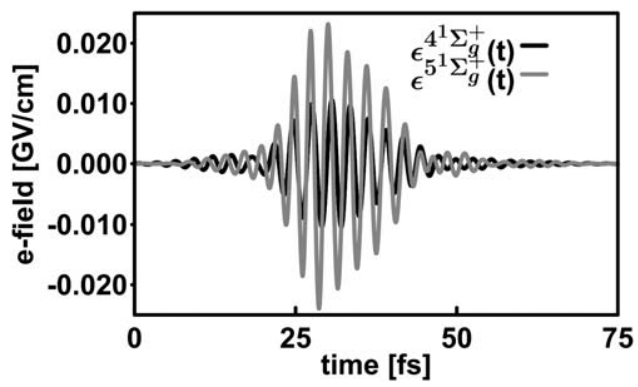

Fig. 10 Comparison of the optimized pulse sequences selectively populating the $4^{1} \Sigma_{\mathrm{g}}^{+}\left(\varepsilon^{4^{4} \Sigma_{\mathrm{g}}^{+}}(t)\right)$ and the $5^{1} \Sigma_{\mathrm{g}}^{+}\left(\varepsilon^{5^{1} \Sigma_{\mathrm{g}}^{+}}(t)\right)$. 
between the two pulses is the intensity. The lower intensity is found foritheAtivitio Online target state in order to suppress the competing transitions in the frequency spectrum of the pulse. The higher intensity for the $5^{1} \Sigma_{\mathrm{g}}^{+}$target state is needed, because the energies of the two target states are not symmetric around twice the energy difference between the $\mathrm{X}^{1} \Sigma_{\mathrm{g}}^{+}$and $\mathrm{A}^{1} \Sigma_{\mathrm{u}}^{+}$(which would be the ideal case for the SPODS scheme). Thus a higher intensity for the $5^{1} \Sigma_{\mathrm{g}}^{+}$target state is needed in order to suppress the other competing transitions in the frequency spectrum.

\section{Conclusion}

In this work we performed OCT calculations to investigate whether the SPODS mechanism working in the strong field regime is included in the search space of the OCT algorithm. Based on these calculations, we can conclude that the SPODS mechanism is included in the search space, and that OCT is able to remarkably increase the efficiency compared to the double pulse sequence presented in Sec. 4. For the optimization of the selective population of the lower lying $4^{1} \Sigma_{\mathrm{g}}^{+}$target state, the algorithm found the SPODS mechanism without any further constraint or additional starting condition as the optimal route. To optimize the selective population of the higher lying $5^{1} \Sigma_{\mathrm{g}}^{+}$target state we only needed the $\frac{\pi}{2}$ phase jump as an additional starting condition, in order to make the electronic state accessible within the initial guess.

In addition our calculations demonstrate that the SPODS mechanism is an optimal solution in the OCT search space. From the properties of the OCT algorithm it is known that high quality control and robust solutions are found even for complex quantum systems including a large number of control variables. ${ }^{31} \mathrm{In}$ this sense the SPODS can be regarded as a robust way to control the selective population of higher lying electronic states, opening a wide spectrum of application ranging form reaction control within molecules up to discrimination between different molecules in a mixture. For larger molecules, holding a more complex electronic structure, the frequency shaped OCT algorithm ${ }^{32}$ might be helpful to optimize the SPODS mechanisms in order to avoid competing resonant transition.

\section{Acknowledgements}

The authors would like to thank Thomas Baumert and Matthias Wollenhaupt for fruitful discussions. We are also grateful for support by the DFG via the Cluster of Excellence: Munich Centre of Advanced Photonics, the SFB749 and the Normalverfahren.

\section{References}

1 T. Brixner and G. Gerber, ChemPhysChem, 2003, 4, 418.

2 F. Krausz and M. Ivanov, Rev. Mod. Phys., 2009, 1, 163.

3 M. F. Kling, C. Siedschlag, A. J. Verhoef, J. I. Khan, M. Schultze, T. Uphues, Y. Ni, M. Uiberacker, M. Drescher, F. Krausz and M. J. J. Vrakking, Science, 2006, 312, 246-248.

4 M. Y. I. B. J. Sussman, D. Townsend and A. Stolow, Science, 2006, 314, 278.

5 E. T. Sleva, I. M. Xavier Jr. and A. H. Zewail, J. Opt. Soc. Am. B, 1986, 3, 483.

6 S. R. Hartmann and E. L. Hahn, Phys. Rev., 1962, 128, 2042.

7 A. D. H. R. Kosloff and D. Tannor, Phys. Rev. Lett., 1992, 69, 2172.

8 R. Netz, T. Feurer, G. Roberts and R. Sauerbrey, Phys. Rev. A: At., Mol., Opt. Phys., 2002, 65, 043406.

9 U. Gaubatz, P. Rudecki, S. Schiemann and K. Bergmann, J. Chem. Phys., 1990, 92, 5363.

10 K. Bergmann, H. Theuer and B. W. Shore, Rev. Mod. Phys., 1998, 70, 1003.

11 M. Wollenhaupt, A. Präkelt, C. Sarpe-Tudoran, D. Liese and T. Baumert, J. Opt. B: Quantum Semiclassical Opt., 2005, 7, S270.

12 M. Wollenhaupt, A. Präkelt, C. Sarpe-Tudoran, D. Liese and T. Baumert, J. Mod. Opt., 2005, 52, 2187. 
13 M. Wollenhaupt, D. Liese, A. Präkelt, C. Sarpe-Tudoran and T. Baumert, Chiet Arthikele Online Lett., 2006, 419, 184.

14 M. Wollenhaupt, A. Präkelt, C. Sarpe-Tudoran, D. Liese and T. Baumert, Appl. Phys. B: Lasers Opt., 2006, 82, 183.

15 M. Wollenhaupt and T. Baumert, J. Photochem. Photobiol., A, 2006, 180, 248.

16 P. von den Hoff, R. Siemering, M. Kowalewski and R. de Vivie-Riedle, IEEE Journal of Selected Topics in Quantum Electronics, 2011, (in press).

17 D. Geppert, P. von den Hoff and R. de Vivie-Riedle, J. Phys. B: At., Mol. Opt. Phys., 2008, 41, 074006.

18 I. Znakovskaya, P. von den Hoff, S. Zherebtsov, A. Wirth, O. Herrwerth, M. Vrakking, R. de Vivie-Riedle and M. Kling, Phys. Rev. Lett., 2009, 103, 103002.

19 P. von den Hoff, I. Znakovskaya, M. Kling and R. de Vivie-Riedle, Chem. Phys., 2009, 366, 139.

20 W. Zhu, J. Botina and H. Rabitz, J. Chem. Phys., 1998, 108, 1953-1963.

21 W. Zhu and H. Rabitz, J. Chem. Phys., 1998, 109, 385-391.

22 D. J. Tannor and S. A. Rice, Adv. Chem. Phys., 1988, 70, 441.

23 C. M. Tesch and R. de Vivie Riedle, Phys. Rev. Lett., 2002, 89, 157901.

24 C. M. Tesch and R. de Vivie-Riedle, J. Chem. Phys., 2004, 121, 12158.

25 R. de Vivie-Riedle, K. Sundermann and M. Motzkus, Faraday Discuss., 1999, 113, 303.

26 K. Sundermann and R. de Vivie-Riedle, J. Chem. Phys., 1999, 110, 1896.

27 J. Manz, K. Sundermann and R. de Vivie-Riedle, Chem. Phys. Lett., 1998, 290, 415-422.

28 J. Somloi, V. A. Kazakov and D. J. Tannor, Chem. Phys., 1993, 172, 85-98.

29 J. P. Palao and R. Kosloff, Phys. Rev. A: At., Mol., Opt. Phys., 2003, 68, 062308.

30 J. C. Diels and W. Rudolph, Ultrashort Laser Pulse Phenomena, Academic Press, Inc., 1996.

31 H. Rabitz, J. Mod. Opt., 2004, 51, 2469.

32 C. Gollub, M. Kowalewski and R. de Vivie-Riedle, Phys. Rev. Lett., 2008, 41, 073002. 